\title{
Efectos en el desarrollo motor de un programa de estimulación motriz basado en actividades lúdicas globalizadas, en varones escolares de la ciudad de Valdivia

\author{
Effects on motor development of a motor stimulation program based on \\ globalized playful activities in male schoolchildren from the city of Valdivia
}

*Eduardo Antonio Mera Massri, *Juan Carlos Armijos Armijos, **Cristian Eduardo Luarte Rocha *Universidad Santo Tomás (Chile), **Universidad San Sebastián (Chile)

Resumen. Objetivo: Analizar los efectos de un programa de estimulación motriz de 8 semanas basado en actividades lúdicas, en el nivel de desarrollo motor grueso de varones escolares de tercero y cuarto básico del Instituto SalesianoValdivia. Método: Participaron 15 alumnos hombres, con una edad de $9.03 \pm 0.63$ años en un programa de estimulación motriz basado en actividades lúdicas, realizando tres sesiones semanales, por un tiempo de ocho semanas. Se midió el nivel de desarrollo motor grueso antes y después de la intervención, utilizando el Test de Desarrollo Motor Grueso (TGMD-2), el cual determina la edad motriz a través de la evaluación de habilidades manipulativas y locomotoras. Resultados: Los resultados mostraron que hubo una diferencia significativa entre pre y post test en las habilidades locomotoras $(p=.0006)$ manipulativas $(p=.0006)$ y el quociente de los resultados ( $p=.0006$ ), pasando de un $93 \%$ de niños bajo edad, pobre y muy pobre a un $93 \%$ de niños en categorías en la edad y sobre la edad. Conclusión: Se concluye que el programa de estimulación motriz basado en actividades lúdicas mejora significativamente los niveles de desarrollo motor en niños.

Palabras clave: Desarrollo Motor, Actividades Lúdicas, TGMD-2.

\begin{abstract}
Objective:To analyze the effects of an 8-week motor stimulation program based on playful activities, in the level of gross motor development of third and fourth grade school boys from the Salesiano Valdivia Institute. Method: 15 male students, with an age of $9.03 \pm 0.63$ years, participated in a motor stimulation program based on recreational activities, performing three weekly sessions, for a period of eight weeks. The level of gross motor development was measured before and after the intervention, using the Test Gross Motor Development (TGMD-2), which determines motor age through the evaluation of manipulative and locomotor skills. Results: The results showed that there was a significant difference between the locomotor skills $(p=.0006)$ and manipulative $(p=.0006)$ and the quoient of the results $(p=.0006)$, going from $93 \%$ of children low age, poor and very poor even $93 \%$ of children in categories in age and over age. Conclusion: It is concluded that the motor stimulation program based on playful activities significantly improves the levels of motor development in children.
\end{abstract} Keywords: Motor development, Playful activities, TDMG-2.

\section{Introducción}

La Organización Mundial de la Salud (OMS), menciona que la actividad física es esencial a lo largo de la vida, como potenciador del crecimiento y desarrollo, y como un agente de prevención para distinto tipo de patologías. Los niños no son una excepción, por lo que es recomendado que realicen actividad física moderada o vigorosa por un mínimo de 60 minutos diarios (OMS, 2020). Dichas actividades, según la recomendación de la OMS deben ser llamativas, pudiendo darse en forma de juegos, deportes, clases de Educación Física o ejercicio planificado. Con base en lo anterior Lubans, Morgan,

Fecha recepción: 21-12-20. Fecha de aceptación: 24-08-21

Eduardo Antonio Mera Massri

mera.massri@gmail.com
Cliff, Barnett \& Okely, (2010) mencionan que el desarrollo temprano de las habilidades fundamentales del movimiento tiene beneficios motores, biológicos y psicológicos. Por otra parte, Burns, Brusseau, Fu, \& Hannon, (2017) mencionan que existe una relación significativa entre las habilidades locomotoras generales y el riesgo cardiometabólico en niños.

Ese planteo, orienta el trabajo hacia otra pregunta: ¿Por qué es importante el desarrollo motor? La competencia motriz favorece y mejora todas las competencias básicas de una persona mediante el desarrollo de las capacidades y habilidades físicas, la utilización del cuerpo como medio de comunicación y expresión y la integración en la vida cotidiana de la actividad física para gozar de buena salud. En otros términos, la conducta motriz implica nada más ni nada menos que la organización significativa del comportamiento motor. Es por ello que su vinculación a la Educación Física Escolar, se 
torna imprescindible (Rijo, Fernández, Hernández, Sosa, $\&$ Pacheco, 2020). De este modo, la asignatura de educación física dispone de un gran repertorio de recursos pedagógicos para promover una educación física integral, destacando entre ellos el juego motor. Es a través del juego motor que se puede potenciar el desarrollo de las competencias motrices y socio-emocionales, y donde el profesor de Educación Física puede desempeñar un papel destacado (Muñoz-Arroyave, Lavega-Burgués, Costes, Damian, \& Serna, 2020).

En consecuencia, es importante el estímulo a través del ejercicio físico en niños, pero se debe considerar que el responsable en guiar la actividad sea una persona capacitada en área. Karim, et al (2015), al igual que Retamal-Valderrama, et al. (2019), recalcan la importancia de que los procesos de formación motora en niños sean liderados por profesionales de la Educación Física, quienes poseen las herramientas para guiarlos en su formación motora.. Con base en el anterior argumento, investigaciones aplicadas en el contexto chileno, muestran que el nivel de desarrollo motor de alumnos en establecimientos educacionales que no cuentan con un profesional de Educación Física, suele ser menores que en instituciones que cuentan con ese profesional, atribuyendo una gran importancia a la cantidad de horas semanales en donde los niños realizan actividad física (Poblete, Morilla \& Quintana, 2015; Luarte, Flores \& Poblete, 2014). No obstante, se comprende y se aclara que otros factores diferentes a la Educación Física (y que no son motivo de esta investigación) también impactan en el desarrollo motor de los niños. Dicha cuestión no es un proceso monocausal.

Es importante mencionar que el requerimiento diario de actividad física en niños, no se cumplirá solo con las pocas horas semanales de Educación Física que realizan los establecimientos chilenos. De acuerdo con el Ministerio de Educación (2020), los Planes de Estudio definen 4 horas de Educación Física de $1^{\circ}$ a $4^{\circ}$ básico y 2 horas desde $5^{\circ}$ básico a IV medio (JEC). Esto implica, dos clases a la semana y una clase a la semana, respectivamente, equivalente a un 8,5\% y 4,25\% del tiempo que están los estudiantes en los establecimientos educacionales. Un estudio reciente (Giakoni, Paredes Bettancourt, \& Duclos-Bastías, 2020) sostiene que en Chile las clases de Educación Física constituyen un espacio de aprendizaje motriz y una instancia idónea para mantener y mejorar el estado de la salud física de los estudiantes escolares. Esto es así ya que comprobaron que el peso, $\mathrm{VO}_{2 \text { máx }} \mathrm{y}$ el nivel de actividad física se ven modificados positivamente en aquellos estudiantes que realizan cuatro horas semanales en vez de dos. Además, concluyen que los hombres son más activos físicamente que las mujeres y presentan mejor condición física.

Luarte, Flores \& Poblete (2012), establecen que con la participación de profesionales de la Educación Física un $34 \%$ de los alumnos evaluados se encuentran con niveles de desarrollo motor en la edad y sobre la edad, mencionando la necesidad de buscar estrategias para generar instancias de acondicionamiento, más allá de las pocas horas de clase en el colegio. Asimismo, Poblete, Flores \& Bustos (2013) evidencian que los alumnos que asisten a talleres extracurriculares manifiestan mejores niveles de desarrollo motor que los alumnos que solo asisten a clases de Educación Física, planteándolo como una necesidad para la correcta formación del alumnado. Reforzando lo planteado, Arufe Giráldez (2019) sostiene que la Educación Física en Educación Infantil concentra un gran protagonismo en el desarrollo óptimo de cada una de las esferas del niño: física, social, afectivoemocional y psíquica. Del mismo modo, entiende que un buen nivel de desarrollo motor es un predictor de buenos niveles de aptitud física. Es por ello que dotar de una gran información y formación a los futuros docentes de Educación Infantil es una pieza clave en el puzle del currículo educativo. Por último, pone foco en la importancia del juego como vehículo generador de aprendizaje del niño.

Por consiguiente, si un niño es guiado por un profesional de manera correcta, es probable que aparte de mejorar su nivel de desarrollo motor pueda verse beneficiado en el desarrollo de factores del aprendizaje (Gil, Contreras, Gómez, 2008; De León, 2013; (Rijo, Fernández, Hernández, Sosa, \& Pacheco, 2020). Con base en esta afirmación, Herazo, Domínguez \& Zota (2010) plantea que el desarrollo motor juega un papel fundamental en la niñez, debido a que el movimiento es un facilitador en el desarrollo intelectual y afectivo, dificultando el desempeño de habilidades complejas en la adolescencia y adultez si es que no se trabaja de buena manera. En ese sentido, Westendorp, Hartman, Houwen, Smith \& Visscher (2011) señalan una relación positiva entre el aprendizaje del lenguaje y el desarrollo de las habilidades locomotoras, y a su vez una relación entre las matemáticas y el desarrollo de las habilidades de control de objeto. En consecuencia, un desarrollo motor acorde a la edad influye positivamente en el desarrollo cognitivo de los niños en formación (Veldman, Santos, Jones, Sousa-Sá \& Okely, 2019).

En base a lo mencionado, un buen nivel de desarro- 
llo motor en la niñez puede traer beneficios por sobre lo motor, pero se deben considerar actividades motivadoras para trabajarlo, de esta manera se genera motivación y entrega hacia la actividad. Es por eso que el presente estudio tiene como objetivo analizar los efectos de un programa de estimulación motriz de 8 semanas basado en actividades lúdicas, en el nivel de desarrollo motor grueso de varones escolares de 9.03 \pm 0.63 años, correspondientes a tercero y cuarto básico del Instituto Salesiano Valdivia. Desde este trabajo, se ha considerado que esa es la edad que mejor se amolda a los objetivos del programa, por tratarse de niños que combinan la inmadurez propia de la edad pero que a su vez disponen suficientes capacidades y aptitudes para aplicar los contenidos que se pretenden inculcar.

El programa a implementar consiste en la aplicación del Test de Desarrollo Motor Grueso - $2^{\text {a }}$ Edición. (TGMD-2), el cual determina la edad motriz a través de la evaluación de habilidades manipulativas y locomotoras. Dicho test, se efectuaría en 24 sesiones, con una frecuencia de tres sesiones por semana, cada una con una duración de 75 minutos. A partir de ello, se podrá determinar si las técnicas empleadas han dado resultados satisfactorios, y en caso de ser así, identificar cuáles han sido esos resultados.

El estudio pretende ser un aporte, actualizado y sostenido tanto en la teoría como en la empírea, de un tema siempre presente y en constante revisión como son técnicas para perfeccionar el desarrollo motor en los niños. Desde un caso de análisis concreto y focalizado, se pretende tomar posición en una temática general.

Para mayor claridad y un mejor desarrollo, el trabajo será distribuido en diferentes apartados: Metodología (participantes, instrumentos, procedimientos y análisis estadísticos), Resultados, Discusión, y las respectivas Conclusiones.

\section{Metodología}

\section{Participantes}

El estudio se llevó a cabo bajo un diseño metodológico cuasi-experimental con muestreo no probabilístico por conveniencia (Hernández, Fernández \& Baptista, 2014). La variable independiente (programa de estimulación motriz basado en actividades lúdicas globalizadas) se manipula para medir sus efectos sobre la variable dependiente (desarrollo motor), con el propósito de determinar si existen cambios significativos producidos por la intervención. Los criterios de inclusión para el estudio fueron: Personas del sexo masculino, pertenecer a los niveles de $3^{\circ}$ o $4^{\circ}$ básico del Instituto Salesiano de Valdivia, no presentar problemas físicos que alteren los resultados, firma de un consentimiento informado aprobado por parte de su tutor, poseer una asistencia mayor o igual al 80\% de las sesiones. Los criterios de exclusión para el estudio fueron: presentar problemas de salud graves o contraindicación médica para realizar ejercicio, asistencia menor al 80\% de las clases, noo presentar consentimiento informado por parte del tutor. La muestra se compone por 15 niños varones de 9.03 \pm 0.63 años, de tercero y cuarto básico, quienes fueron inscritos en el taller de atletismo del Instituto SalesianoValdivia. Se trata de un grupo único y reducido ya que, si bien simplifica el análisis, trabajar con otros grupos para un mayor control complejizaría en demasía el logro de los objetivos propuestos, mientras que una muestra con número de alumnos más elevado impactaría negativamente en la calidad del trabajo con los niños y por sobre todo en su evaluación.

Para llevar a efecto el estudio, se realizó una reunión con los apoderados de cada alumno, los cuales fueron informados sobre el programa, instrumentos y procedimientos para aplicar en el estudio, así como riesgos y beneficios que podrían presentarse, avalando mediante carta de consentimiento la participación de su pupilo. El asentimiento de padres (o tutores) fue del 100\%. Se evaluaron a todos los participantes que mantuvieron una asistencia mayor o igual al 80\% de las sesiones.

De otro lado, la intervención del docente, en líneas generales, funcionó como guía, orientador y artífice principal para que la actividad física se desarrolle positivamente y con normalidad. Más específicamente, en primer término, realizó la explicación teórica en forma oral de la actividad del día, explicitando sus reglas, los límites y respondiendo preguntas o dudas de los alumnos. Luego en la introducción, dirigió los movimientos precompetitivos y brindó la explicación en forma técnica o práctica de la actividad. Durante el desarrollo, supervisó de cerca la actividad principal y ofició como juez o árbitro ante cualquier litigio. El objetivo fue el desarrollo sin interrupciones u obstáculos de la actividad planeada. Por último, finalizó la actividad y se convirtió en un observador en el desenlace, adquiriendo un rol pasivo en el cual observó y tomó nota de cómo cada alumno utiliza su tiempo libre.

\section{Instrumentos}

Se utilizó el Test of Gross Motor Development - $2^{\text {a }}$ edition (TGMD-2) (Ulrich, 2000). Este instrumento determina el nivel de desarrollo motor mediante habi- 
lidades locomotrices (HL) y habilidades de control de objetos (HCO). Las HL se miden a través de seis pruebas: correr, galopar, saltar en un pie, saltar un obstáculo con carrera previa, saltar horizontalmente a pies juntos y, deslizamiento lateral sin desviarse. Las HCO se miden a través de otras seis pruebas: batear una pelota estática, botar un balón de baloncesto con la mano dominante, recepción de una pelota con las dos manos, chutar un balón estático, lanzar una pelota con mano dominante y, lanzar rodando una pelota entre dos conos. Cada habilidad se mide a partir de tres a cuatro criterios de rendimiento cualitativo en base al patrón motor referido, constituyendo el objeto de la evaluación. De la sumatoria de la evaluación de los test para cada habilidad se establece un puntaje, el cual depende tanto de la evaluación como de la edad del participante. Con el puntaje para cada habilidad se puede establecer la edad de esta, y con la sumatoria de estos puntajes se calcula un cociente, el cual es necesario para establecer si el nivel de desarrollo motor del evaluado se encuentra en categorías sobre la edad, bajo la edad o en la edad.

Para administrar el test es necesario el siguiente material: un balón de 20 a $25 \mathrm{~cm}$, una pelota de $10 \mathrm{~cm}$, un balón de baloncesto, una pelota de tenis, un balón de fútbol, una pelota de softball, un saquito de 10 a $12 \mathrm{~cm}$, dos conos, un tee de bateo y cinta adhesiva. El test de Ulrich fue validado por Cano-Cappellacci, Leyton y Carreño (2015) para ser utilizado en Chile.

Tabla 1.

\begin{tabular}{|c|c|c|c|}
\hline Sesiones & Introducción (15min) & Desarrollo (45 min) & Final $(15 \mathrm{~min})$ \\
\hline $\begin{array}{c}\text { Semana } 1 \\
\text { (Sesión } 1 \text { a } 3 \text { ) }\end{array}$ & $\begin{array}{l}\text { Basada en habilidades } \\
\text { locomotoras de correr, } \\
\text { deslizamiento y galopa. }\end{array}$ & $\begin{array}{l}\text { Trabajo en base a juegos o } \\
\text { desafíos, orientado a la habilidad } \\
\text { de correr, galopa y deslizamiento. } \\
\text { Actividades relacionadas al } \\
\text { atletismo. }\end{array}$ & $\begin{array}{l}\text { Los alumnos realizan } \\
\text { actividades libres con el } \\
\text { material disponible de } \\
\text { la sesión. }\end{array}$ \\
\hline $\begin{array}{c}\text { Semana } 2 \\
\text { (Sesión 4 a 6) }\end{array}$ & $\begin{array}{l}\text { Basada en habilidades } \\
\text { locomotoras de saltos } \\
\text { (a dos pies, en un pie y } \\
\text { por sobre objetos). }\end{array}$ & $\begin{array}{l}\text { Trabajo en base a juegos o } \\
\text { desafíos, orientado a la habilidad } \\
\text { de saltos. Actividades relacionadas } \\
\text { al atletismo. }\end{array}$ & $\begin{array}{l}\text { Los alumnos realizan } \\
\text { actividades libres con el } \\
\text { material disponible de } \\
\text { la sesión. }\end{array}$ \\
\hline $\begin{array}{c}\text { Semana } 3 \\
\text { (Sesión 7 a 9) }\end{array}$ & $\begin{array}{l}\text { Basada en habilidad } \\
\text { manipulativa de drible. }\end{array}$ & $\begin{array}{l}\text { Trabajo en base a juegos o } \\
\text { desafíos, orientado a la habilidad } \\
\text { de drible. Actividades } \\
\text { relacionadas al atletismo. }\end{array}$ & $\begin{array}{l}\text { Los alumnos realizan } \\
\text { actividades libres con el } \\
\text { material disponible de } \\
\text { la sesión. }\end{array}$ \\
\hline $\begin{array}{c}\text { Semana } 4 \\
\text { (Sesión 10 a 12) }\end{array}$ & $\begin{array}{l}\text { Basada en habilidad } \\
\text { ) manipulativa de bateo. }\end{array}$ & $\begin{array}{l}\text { juegos o } \\
\text { lo a la habilidad } \\
\text { na. Actividades } \\
\text { letismo. } \\
\end{array}$ & $\begin{array}{l}\text { Los alumnos realizan } \\
\text { actividades libres con el } \\
\text { material disponible de } \\
\text { la sesión. }\end{array}$ \\
\hline $\begin{array}{l}\text { Semana } 5 \\
\text { (Sesión 13 a 15) }\end{array}$ & $\begin{array}{l}\text { Basada en habilidad } \\
\text { ) manipulativa de lanzar }\end{array}$ & $\begin{array}{l}\text { Trabajo en base a juegos o } \\
\text { desafíos, orientado a la habilidad } \\
\text { de lanzar. Actividades relacionadas } \\
\text { al atletismo. }\end{array}$ & $\begin{array}{l}\text { Los alumnos realizan } \\
\text { actividades libres con el } \\
\text { smaterial disponible de } \\
\text { la sesión. }\end{array}$ \\
\hline $\begin{array}{c}\text { Semana } 6 \\
\text { (Sesión } 16 \text { a 18) }\end{array}$ & $\begin{array}{l}\text { Basada en habilidad } \\
\text { manipulativa de } \\
\text { recepción. }\end{array}$ & $\begin{array}{l}\text { Trabajo en base a juegos o } \\
\text { desafíos, orientado a la habilidad } \\
\text { de recepción. Actividades } \\
\text { relacionadas al atletismo. }\end{array}$ & $\begin{array}{l}\text { Los alumnos realizan } \\
\text { actividades libres con el } \\
\text { material disponible de } \\
\text { la sesión. }\end{array}$ \\
\hline $\begin{array}{c}\text { Semana } 7 \\
\text { (Sesión 19 a 21) }\end{array}$ & $\begin{array}{l}\text { Basada en habilidad } \\
\text { manipulativa de } \\
\text { golpear. }\end{array}$ & $\begin{array}{l}\text { Trabajo en base a juegos o } \\
\text { desafíos, orientado a la habilidad } \\
\text { de golpear. Actividades } \\
\text { relacionadas al atletismo. }\end{array}$ & $\begin{array}{l}\text { Los alumnos realizan } \\
\text { actividades libres con el } \\
\text { material disponible de } \\
\text { la sesión. }\end{array}$ \\
\hline $\begin{array}{c}\text { Semana } 8 \\
\text { (Sesión } 22 \text { a 24) }\end{array}$ & $\begin{array}{l}\text { Basada en habilidad } \\
\text { manipulativa de rodar. }\end{array}$ & $\begin{array}{l}\text { Trabajo en base a juegos o } \\
\text { desafíos, orientado a la habilidad } \\
\text { de rodar. Actividades relacionadas } \\
\text { al atletismo. }\end{array}$ & $\begin{array}{l}\text { Los alumnos realizan } \\
\text { actividades libres con el } \\
\text { material disponible de } \\
\text { la sesión. }\end{array}$ \\
\hline
\end{tabular}

\section{Procedimientos}

La intervención consta de un programa de ejercicios basado en actividades lúdicas. El programa se constituyó de un total de 24 sesiones, con una frecuencia de tres sesiones por semana, con una duración de 75 minutos por sesión (ver tabla 1). El tiempo se distribuyó en términos tentativos o aproximados del siguiente modo: 15 minutos para la introducción, 45 minutos para el desarrollo de la actividad principal, y los restantes 15 minutos para el desenlace. Las sesiones fueron realizadas dentro de las instalaciones deportivas del Instituto Salesiano Valdivia. Se aplicó el TGMD2 la semana antes de iniciar la intervención y la semana posterior a la última sesión realizada.

\section{Análisis estadístico}

Para la presente investigación se procedió a realizar un análisis de datos utilizando el programa estadístico Stata V.12.0. En primera instancia se realizó un análisis de tipo descriptivo y posteriormente inferencial. En la estadística descriptiva se presentaron el número total de sujetos, desviación estándar, percentil 25, 50 y 75 y las medidas de tendencia central como promedio, mediana, mínima y máxima. La normalidad de los datos se verificó por medio del test Shapiro-Wilk. La distribución de las variables fue no normal, por tanto, se utilizó el test de Wilcoxon. Se determinó $p<0.05$ para establecer diferencias significativas entre los datos.

\section{Resultados}

A continuación, se presentan los resultados obtenidos tras la aplicación del test de Ulrich en forma de tablas y figuras.

La Tabla 2 muestra la clasificación general del TGMD2, donde se clasifica a los participantes en base a la relación de su edad motora respecto a la edad biológica. En base a los resultados del pre-test, la edad motora de un 93\% de los participantes presentaba un nivel de desarrollo motor era menor a su edad cronológica, mientras que solo una persona se encontraba en concordancia de edad. En el post-test, 47\% de los participantes presenta un nivel de desarrollo motor superior a la edad cronológica, al igual que un 47\% de los participantes se encuentra en concordancia con la edad. Solo un participante del estudio está en una categoría pobre.

La tabla numero 3 enseña la relación entre variables a través del test de Wilcoxon para los resultados del test locomotor, manipulación de objetos y el quociente. Se indica que hubo diferencias significativas para las tres 
variables respecto a los resultados del TGMD2 pre y post intervención.

La tabla numero 4 muestra los totales de los puntajes del test locomotor, de control de objetos y quociente pre y post intervención. Se aprecia una mejora en los puntajes de los participantes del estudio.

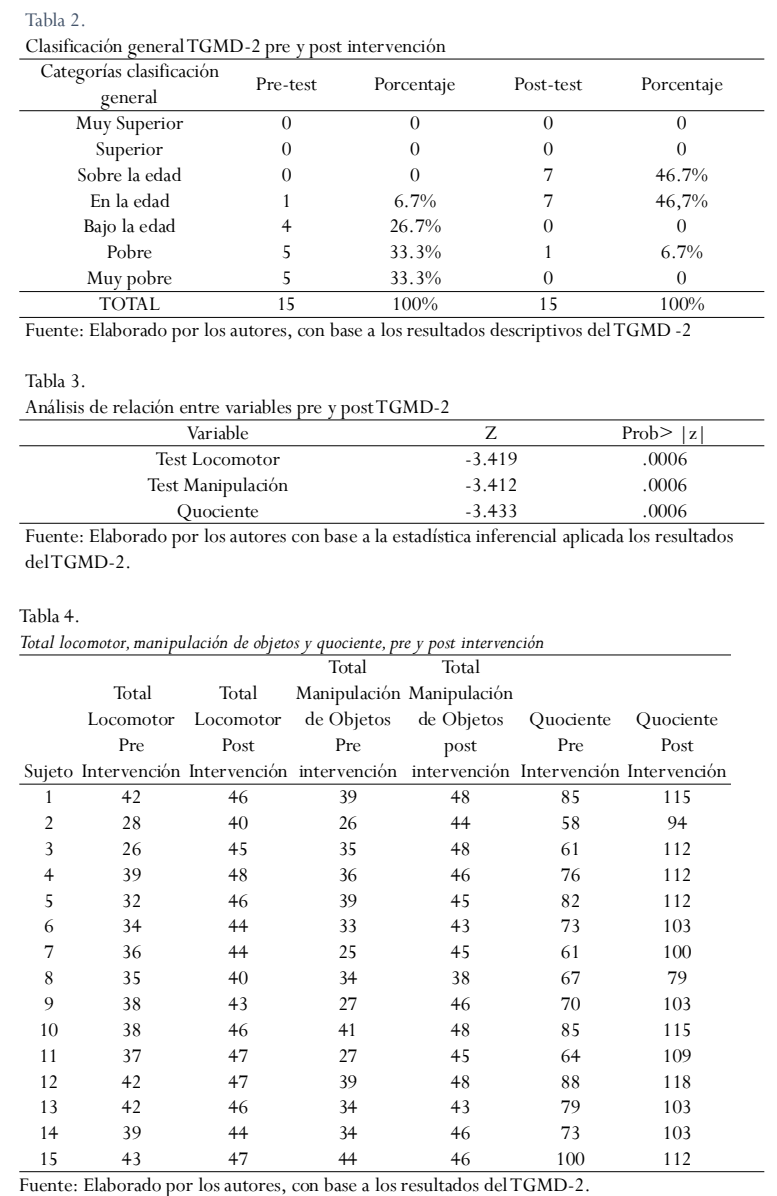

La figura 1 refleja la puntuación del test locomotor pre y post intervención. Se aprecia una mejora en post intervención al igual que una disminución en la dispersión de datos. La tabla 3 refleja que las mejoras post intervención son significativas.

La figura 2 refleja la puntuación del total de la prueba de control de objetos pre y post intervención. Post intervención la dispersión de datos disminuyo centrándose en puntajes mayores. La tabla 3 refleja que las mejoras post intervención son significativas.

La figura 3 refleja la puntuación del total del quociente pre y post intervención. Se aprecia que post intervención la dispersión de datos disminuyo teniendo como media un puntaje mayor, esta mejora es significativa según los resultados de la tabla 3. Todos los resultados expresados en las distintas graficas mejoraron a tal punto de estar por sobre la media de los test aplicados antes de la intervención.

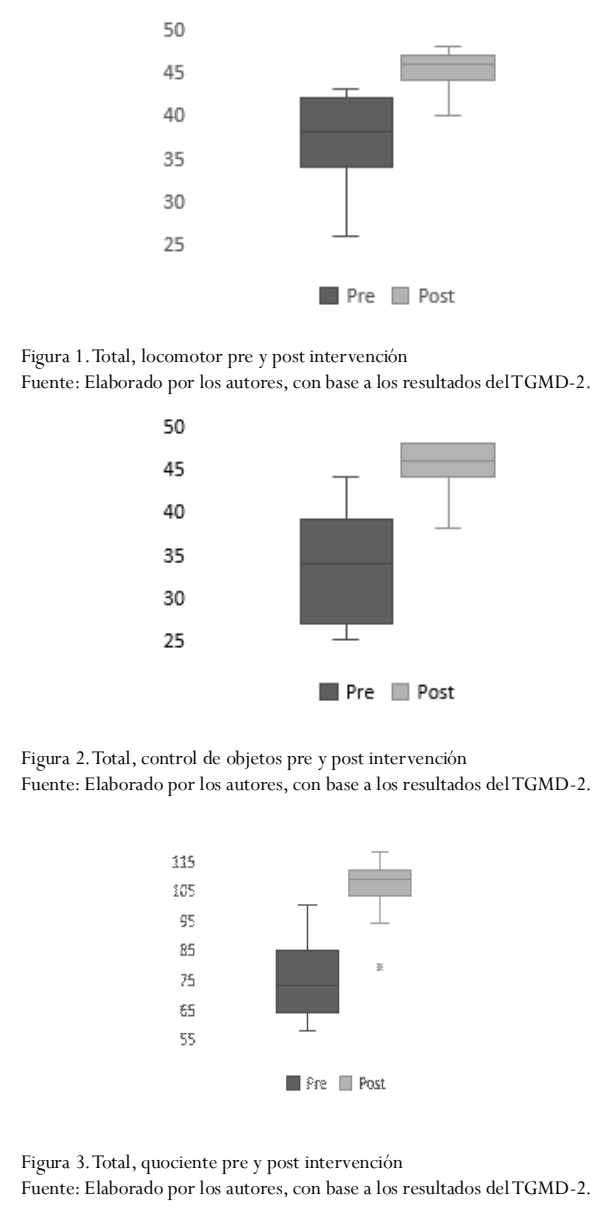

\section{Discusión}

El resultado principal del estudio demuestra que un programa de estimulación motriz basado en actividades lúdicas, mejora significativamente los niveles de desarrollo motor. En este trabajo, se da por sentado que el deporte y los juegos implementados en la clase de educación física a través de un profesor que planifica las clases generan satisfacción en los alumnos. Un estudio (entre tantos otros, Gråstén, Jaakkola, Liukkonen, Watt, \& Yli-Piipari, 2012; Rodríguez-Rodríguez, Curilem, Berral, \& Flores, 2017) que da prueba de ello es el llevado adelante por Zueck, Ramírez, Rodríguez e Irigoyen (2019) quienes demuestran la gran influencia de la clase de educación física en los alumnos del nivel de primaria en sus niveles de satisfacción e intención a la práctica física. Esa investigación obtiene dos grandes conclusiones: que los niveles de satisfacción son mayores cuando las clases son debidamente planeadas, y de igual modo es mayor cuando se practican deporte en comparación con otras actividades.

Volviendo a este estudio, en base a los antecedentes mencionados, no se encontró necesario medir los niveles de satisfacción, lo cual hubiese demandado mayor 
tiempo, dedicación y otros medios para llevar adelante el proyecto. También se tiene como punto de partida que se trabajó con un único grupo y reducido en el cual un requisito de inclusión fue alumnos que explícitamente les agrada el deporte y se interesan por las clases de educación Física (aunque tampoco se trato de un concurso selectivo y excluyente). En consecuencia, se dio por probado que el nivel de disfrute sería alto o al menos considerable para realizar el trabajo.

En consecuencia, esta investigación se encuentra en concordancia con diversos autores que plantean programas de ejercicio para la mejora del desarrollo motor, Rudd, Barnett, Farrow, Berry, Borkoles \& Polman (2016), obtuvieron mejoras significativas en la estabilidad y habilidades de control de objetos, aplicando un programa de ejercicio basado en la gimnasia con una sesión semanal de dos horas por 16 semanas. Otros autores (Yildirim, Bilge, \& Caglar, 2019; Piña, Ochoa, Hall, Reyes, Alarcón, Monreal, \& Sáenz-López, 2020) también han obtenido mejoras del desarrollo motor a través de intervenciones ligadas al ejercicio físico, como por ejemplo por medio del deporte, tanto de equipo (baloncesto, fútbol, balonmano y voleibol) como individuales (deportes de raqueta y deportes de contacto). Yildirim et al. (2019) trabajó con grupos que realizaban distintos tipos de ejercicio en sesiones de 90 minutos, tres días a la semana, por seis meses, obteniendo mejoras significativas en los resultados del TGMD2. Piña et al. (2020) aplicando un programa de ejercicio por 12 semanas con tres sesiones semanales, obtuvo mejoras significativas en la motricidad gruesa y fina. Por otra parte, Frith, \& Loprinzi, (2019) evidencian que un buen nivel de habilidad motora se asocia a un buen nivel de resistencia musculoesquelética en niños. Reforzando lo anterior Wick et al. (2017) concluyen en su meta-análisis que existe una efectividad relevante en los programas de intervención para mejorar las habilidades fundamentales del movimiento en niños pequeños sanos.

En Chile se imparten pocas horas de Educación Física semanales, lo que no es suficiente en caso de que quisieran replicar el tiempo de intervención aplicado en esta investigación. El único medio por el que podrían aplicarlo sería a través de instancias extracurriculares, donde ya se ha evidenciado de que existen diferencias significativas en los niveles de desarrollo motor entre alumnos que solo realizan la clase de Educación Física y los que además realizan talleres extracurriculares (Poblete et al., 2013; Pradenas, Campos, Contreras, Puentes, \& Luna, 2017). Los resultados del test previo a la intervención muestran que un 93\% de los partici- pantes se encontraban en niveles de desarrollo motor bajo la edad, pobre o muy pobre, evidenciando una deficiente formación motora para la edad. Esta condición de deficiencia aparece en otras investigaciones de la población escolar, (Poblete et al., 2015; Luarte, et al., 2014) los cuales demostraron que más de 50\% de los alumnos que solo realizan las clases de Educación Física evidencian niveles de desarrollo motor bajo la edad, lo que evidencia una falta de estímulos motrices que potencie un desarrollo saludable en escolares. En base a lo anterior Utesch, Bardid, Büsch, \& Strauss, (2019) recalcan en su meta-análisis que se debe orientar el trabajo de las competencias motoras como el de la aptitud física desde la infancia en adelante, debido a que revelaron una relación positiva entre estas cualidades, lo cual beneficia un crecimiento saludable.

En la presente investigación se realizó un programa de estimulación motriz basado en actividades lúdicas, teniendo mejoras significativas en el test locomotor, de manipulación de objetos y el quociente de los puntajes. En Chile se han aplicado algunos estudios con actividades lúdicas como método de intervención en niños, Fritz \& Vargas (2013) aplicando un programa de actividades lúdicas obtuvieron mejoras en cociente de las habilidades motoras y recalcaron la importancia de las actividades lúdicas, como factor que favorece la participación de los alumnos. Siguiendo la misma línea Luarte, Poblete \& Fuentes (2014) aplicaron un programa de ejercicio basado en actividades lúdicas en un taller de hockey patín, utilizando una estrategia de enseñanza lúdica en un deporte, lo que tuvo como resultado la obtención de mejoras en las categorías de nivel de desarrollo motor, defendiendo el punto que las actividades aplicadas aportan al factor de la motivación de los niños. En estudios más recientes, Luna, Aravena, Contreras, Fabres, \& Faúndez (2016) aplicaron un programa basado en juegos en establecimientos educacionales, obteniendo mejoras significativas en el nivel de desarrollo motor a diferencia del grupo control. Los anteriores resultados concuerdan con lo que plantea Blázquez (1995), quien menciona que actividades deportivas extracurriculares con enfoque pedagógico facilitan el aprendizaje motor, en especial si se producen en un ambiente lúdico. Se debe tener en cuenta que las etapas fundamentales para la estimulación del desarrollo motor se encuentran en la niñez, por ende, es importante que estas actividades sean llamativas y motivadoras siendo que los niños necesitan un ambiente donde puedan realizar actividades lúdicas, debido a que es su esencia (Huizinga, 2012). El objetivo al aplicar actividades lúdicas es generar una 
motivación intrínseca, esto quiere decir que la persona genera un apego hacia la actividad generado por las experiencias estimulantes, esto depende del desenvolvimiento del profesional de Educación Física (Ruiz, Baños, Fuentesal, García \& Baena, 2019). En base a lo anterior debería existir mayor preocupación en las escuelas por realizar actividades más allá de estar en un salón de clase.

Cabe destacar que los participantes de esta investigación son solo varones, de manera tal que la investigación se hace más específica, esto se pudo gracias a que la modalidad del colegio intervenido solo trabaja con alumnos de ese género, lo que en un futuro no va a ser fácil de replicar en este tipo de instituciones debido a que la tendencia es a entregar servicios de educación mixtos, quedando solo dos colegios que tienen pensado en un futuro cambiar a esta modalidad.

El programa ejecutado en esta intervención fue creado por un profesor de educación física que planificó cada sesión en base a las circunstancias de la investigación, adaptando las sesiones a la realidad del establecimiento y los estudiantes intervenidos, por lo que es importante tener en cuenta que la intervención se puede replicar, modificar o adaptar a otros deportes siempre y cuando sea realizado por un profesor de educación física, el cual está capacitado para realizar la labor, de lo contrario es probable que la intervención se vea comprometida.

Cualidades como el manejo de grupo, la resolución de problemas y la capacidad de adecuarse a los tiempos de las sesiones, fueron cruciales para el éxito de la intervención, por el hecho de ser parte de las competencias necesarias de un profesor de Educación Física, siendo cruciales para que los juegos planificados funcionen de la forma esperada.

Hasta el día de hoy existe un registro muy limitado de investigaciones en Chile referente al desarrollo motor, lo que es aún menor cuando se incluyen investigaciones que proponen un programa de intervención para mejorar los niveles en niños. En base a lo anterior, Pavez et al. (2020) realizan una revisión sistemática de artículos científicos que aplicaron test de desarrollo motor, entre los años 2014-2018 con población chilena, obteniendo solo 9 artículos para este rango de tiempo, lo que es un resultado muy pobre considerando la gran cantidad de asociaciones que se puede realizar a partir del tema.

La intervención del docente en la actividad física propuesta fue esencial para que la misma se desarrollara positivamente y con normalidad favoreciendo el aprendizaje en un contexto de disfrute y recreación para los alumnos. A nivel macro, el docente funcionó como guía, orientador y artífice o promotor de los ejercicios físicos. En el plano micro, brindó explicaciones tanto teóricas como prácticas, supervisó las actividades centrales y observó los comportamientos. Corrigió allá donde lo encontró necesario. A lo largo del trayecto, alternó el rol de docente, referente o juez y de evaluador.

Finalmente, no existe un registro con el cual se pueda determinar el nivel de desarrollo motor de la población chilena, pensando en que aparte del bajo número de investigaciones, la gran mayoría se centran en la zona centro sur del país, habiendo un desconocimiento de la población de la zona norte. Es importante el generar aportes al tema de estudio, con el fin de contribuir con el desarrollo del área en el país, teniendo en cuenta que el tema tiene relación tanto con la salud de las personas como con el aprendizaje (Herazo et al., 2010; Westendorp et al., 2011; Veldman et al., 2019). Se espera que la investigación realizada sea parte de estudios mayores, siendo un aporte para el desarrollo de nuevas aplicaciones en el área.

Se espera que el programa de intervención pueda ser usado como modelo (o al menos como referencia) para otras investigaciones del área o como una guía para mejorar los déficits en los niveles de desarrollo motor en niños. Además, se espera que el aplicar actividades lúdicas en niños sea una estrategia de formación utilizada con mayor frecuencia, pensando en que abarca aspectos biopsicosociales.

Como limitación del estudio se establece no contar con un grupo control, no tener previo conocimiento en los antecedentes de actividad física de los preescolares y no tener datos de la composición corporal de los participantes. En el futuro sería importante realizar un número mayor de estudios que clarifiquen situaciones causa efecto respecto a la motricidad de niños de edad preescolar, en diferentes ambientes pedagógicos y contextos sociales.

\section{Conclusiones}

La investigación tuvo como objetivo, analizar los efectos de un programa de estimulación motriz de ocho semanas basado en actividades lúdicas, en el nivel de desarrollo motor grueso de varones escolares de tercero y cuarto básico del Instituto Salesiano Valdivia. El análisis de datos evidencio que los efectos del programa fueron:

Aumento significativo de las habilidades locomo- 
toras de los participantes.

- Aumento significativo de las habilidades de control de objetos de los participantes.

- Aumento significativo del quociente del puntaje de los participantes.

- El desarrollo motor de los participantes paso de ser un 93\% en categorías bajas, a un 93\% en categorías en la edad y sobre la edad.

En base a todo lo anterior, podemos afirmar que la hipótesis de la investigación se cumple, sosteniendo que un programa de estimulación motriz de ocho semanas basado en actividades lúdicas mejora significativamente el nivel de desarrollo motor grueso en varones escolares de tercero y cuarto básico del Instituto Salesiano de Valdivia.

Finalmente, se concluye que un programa de estimulación motriz basado en actividades lúdicas de ocho semanas de duración con tres sesiones semanales, mejora significativamente el nivel de desarrollo motor grueso en varones con una edad de $9.03 \pm 0.63$ años.

\section{Referencias}

Arufe Giráldez,V.(2019).¿Cómodebeser el trabajode Educación Física en Educación Infantil? (¿How should Physical Education work in Early Childhood Education be?). Retos. NuevasTendencias en Educación Física, Deporte y Recreación, 37. pp. 588-596. https://doi.org/10.47197/retos.v37i37.74177

Blázquez, D. (1995). La iniciación deportiva yel deporte escolar. Barcelona: INDE.

Burns, R. D., Brusseau,T.A., Fu,Y., \& Hannon, J.C. (2017). Gross Motor Skills and Cardiometabolic Risk in Children: A Mediation Analysis. Medicine and science in sports and exercise, 49(4), 746-751. https://doi.org/10.1249/ MSS.0000000000001147

Cano-Cappellacci, M. Leyton, F.A. Carreño, J.D. (2015). Content validity and reliability of test of gross motor development in Chilean children. Revista Saude Publica. pp.49:97.

De León, L. (2013). Rol docente inicial en el desarrollo fisico y social del niño de primera infancia.Tesis de Pedagogía con Orientación enAdministración y Evaluación Educativas. Universidad Rafael Landívar. Facultad de Humanidades, Campus de Quetzaltenango.

Frith, E., \& Loprinzi, P. D. (2019). Association Between Motor Skills and Musculoskeletal Physical Fitness Among Preschoolers. Maternal and child health journal, 23(8), 10031007. https://doi.org/10.1007/s10995-019-02753-0

Fritz, J. \& Vargas, R. (2013). Impacto de actividades lúdicas no competitivas sobre las habilidadesmotoras gruesas en niños y niñas pre escolares. Revista Ciencias de la Actividad Física UCM, 14(1)31-37.http://repositorio.ucm.d//bitstream/handle/ $\begin{array}{lllllllll}\mathrm{u} & \mathrm{c} & \mathrm{m} & / & 1 & 3 & 8 & 1\end{array}$ vargas_r_impacto.pdf?sequence $=1$ \&isAllowed $=\mathrm{y}$

Gatica, P.,Vargas, R. y cols. (2002). Evaluación del desarrollo motor en escolares de 6 a 10 años de la región del Maule. Talca: UCM-IND.

Giakoni, F., Paredes Bettancourt, P., \& Duclos-Bastías, D. (2020). Educación Física en Chile: tiempo de dedicación y su influencia en la condición física, composición corporal y nivel de actividad física en escolares (Physical Education in Chile: time spent and its influence on physical condition, body composition, and l. Retos. NuevasTendencias en Educación Física, Deporte y Recreación, 39. pp. 24-29. https://doi.org/ 10.47197/retos.v0i39.77781

Gil, P. Contreras, O. Gómez, I. (2008). Habilidades motrices enla infancia y su desarrollo desde una educación física animada. Revista Iberoamericana de Educación. N. ․ 47.pp. 71-96.https: / /rieoei.org/historico/documentos/rie47a04.pdf

Gråstén, A., Jaakkola, T., Liukkonen, J., Watt, A., \&Yli-Pïpari, S. (2012). Prediction of enjoyment in shool physical education. Journal of Sports Science and Medicine, 11.pp.260-269.

Herazo,Y., Dominguez, R. \&,Zota I.(2010). Estadios delos patrones motores fundamentales en una escuela regular. Fisioterapia, 32(2), 66-72. https: / / doi.org/10.1016/j.ft.2009.11.005

Hernández, R., Fernández, C., \& Baptista, L. (2014). Metodologíade la investigación. México: McGraw-Hill Education.

Huizinga, J.(2012). Homo Ludens. Madrid:Alianza.

Karim, O.A.,Ammar,A., Chtourou, H.,Wagner, M., Schlenker, L., Parish, A., ... \& Bös, K. (2015). A comparative study of physical fitness among Egyptian and German children aged between 6 and 10 years. Advances in Physical Education, 5(1), 7 17. http://dx.doi.org/10.4236/ape.2015.51002

Luarte, C., Flores, C. \& Poblete, F. (2012). Nivel de desarrollo motor grueso en escolares de NB2 deTalcahuano, octava Región, Chile. Revista Ciencias de la Actividad Física UCM, 13, 5157. https://www.redalyc.org/pdf/5256/ 525657739008.pdf

Luarte, C., Poblete, F. \& Flores, C. (2014). Nivel de desarrollo motor grueso en preescolares sin intervención de profesores de Educación Física, Concepción, Chile. Revista Ciencias de la Actividad Fisica UCM, 15(1), 7-16. http: / / revistacaf.ucm.cl/ article/view/36

Luarte, C., Poblete, F. \& Fuentes Montero, G., (2014). Efectos de un programa basado en actividades lúdico-motrices en los niveles de desarrollo motor grueso en niños y niñas de la rama de hockey césped. Rev. horiz., cienc. act. fis. 5, 37-44. http:/ / revistahorizonte.ulagos.cl/index.php/horizonte/article/ view/56

Lubans, D. R., Morgan, P.J., Cliff, D. P., Barnett, L. M., \& Okely,A. D. (2010). Fundamental movement skills in children and adolescents: review of associated health benefits. Sports medicine (Auckland, N.Z.), 40(12), 1019-1035. https:/ /doi.org/ 10.2165/11536850-000000000-00000 
Luna,P.,Aravena,J., Contreras, D., Fabres, C.\& Faúndez,F.(2016). Efectos en el desarrollomotor de un programa de estimulación de habilidades motrices básicas en escolares de $5^{\circ}$ año Básico de colegios particulares subvencionados de Concepción. Revista Ciencias de la Actividad Física UCM, 17(1), 29-38. http:/ / revistacaf.ucm.cl/article/view/88

Ministerio de Educación (MINEDUC). (2020). Orientacionespara la actividad fisica escolar. Unidad de Actividad Física Escolar. https:// convivenciaescolar.mineduc.cl/wp-content/ uploads/2020/11/ORIENTACIONES-ED-FISICAWEB.pdf

Muñoz-Arroyave,V.,Lavega-Burgués,P., Costes,A., Damian,S., \& Serna,J.(2020). Los juegos motores como recursopedagógico para favorecer la afectividad desde la educación física (Traditional games: a pedagogical tool to foster affectivity in physical education). Retos. NuevasTendencias en Educación Física, Deporte y Recreación, 38. pp.166-172. https://doi.org/ 10.47197/retos.v38i38.76556

Organización Mundial de la Salud [OMS]. (2020). Actividad fisica. https://www.who.int/es/news-room/fact-sheets/detail/ physical-activityPavez, G., Hernández, C., Torres, S.,Paillacar, M., Concha, C., Cabrera, M., . . \& \& Gómez, N. (2020).Testde desarrollo motor aplicados en Chile entre el período 20142018. Una revisión sistemática. Revista Ciencias de la Actividad Física UCM, 21(1), 1-13. http://doi.org/10.29035/ rcaf.21.1.1

Piña,D., Ochoa,P., Hall,J., Reyes,Z.,Alarcón,E., Monreal, L., \& Sáenz-López,P. (2020). Efecto de un programa deEducación Física con intensidad moderada vigorosa sobre el desarrollo motor enniñosdepreescolar. Retos, 38(38), 363-368.https:/ /doi.org/10.47197/retos.v38i38.73818

Poblete, F., Flores, C.\& Bustos, S. (2013). Desarrollomotor grueso en alumnos de 8, 9 y 10 años de edad en clases de Educación Física y talleres extracurriculares. Revista de Ciencias de la Actividad Física UCM, 14(2), 21-30. https://doi.org/ 10.29035/rcaf.18.2.1

Poblete, F., Morilla, C., Quintana, C. (2015). Nivelde desarrollo motor grueso en pre-escolares sin intervención de profesores de Educación Física, Valdivia. Rev. horiz., cienc. act. fis. 6, 33-40. http://revistahorizonte.ulagos.cl/index.php/horizonte/ article/view/81

Pradenas, X., Campos, M., Contreras, M., Puentes, D. \& Luna, P. (2017). Comparación del desarrollo motor en escolares de 9 y 10 años deedad en clases de educación física y talleres deportivos extracurriculares. Revista Ciencias De La Actividad Física UCM, 18(2), 1-8. https://doi.org/10.29035/rcaf.18.2.1

Retamal-Valderrama, C., Delgado-Floody,P.,Espinoza-Silva,M., \& Jerez-Mayorga, D. (2019). Comportamiento del Profesor, Intensidad yTiempo Efectivo de las Clases deEducación Física en una escuela pública: Un acercamiento a la realidad. Retos: nuevastendenciasen educación fisica, deporteyrecreación, 35.pp.160163.
Rijo,A.,Fernández,J., Hernández,J.,Sosa, G., \& Pacheco,J.(2020). (Re) pensar la competencia motriz ( $(\mathrm{Re})$ think motor competence). Retos. Nuevas Tendencias en Educación Física, Deporte y Recreación, 40. pp. 375-384. https://doi.org/ 10.47197/retos.v1i40.82959

Rodríguez-Rodríguez,F,CCurilem, C., Berral,F.,\& Flores,A.(2017). Evaluación de la educación física escolar en Enseñanza Secundaria. Retos. Nuevas Tendencias en Educación Física, Deporte y Recreación, 31.pp. 76-81.

Rudd, J., Barnett,L., Farrow, D., Berry, J., Borkoles, E. \& Polman, R. (2016). Effectiveness of a 16 week gymnastics curriculum at developing movement competence in children. Journal of science and medicine in sport, 20(2), 164-169 .10.1016/ j.jsams.2016.06.013

Ruiz, F., Baños, R., Fuentesal, J., García, E. \& Baena,A. (2019). Análisis transcultural del clima motivacional en alumnado de Costa Rica, México y España. Revista Internacional de Medicina y Ciencias de la Actividad Física y el Deporte, 19(74), 351-369. http://doi.org/10.15366/rimcafd2019.74.011

Ulrich, D.A. (2000). The test of gross motor development (2ndEd.). Austin,TX: PROED Publishers.

Utesch, T., Bardid, F., Büsch, D., \& Strauss, B. (2019). The Relationship Between Motor Competence and Physical Fitness from Early Childhood to Early Adulthood:A Meta-Analysis. Sports medicine (Auckland, N.Z.), 49(4), 541-551. https:// doi.org/10.1007/s40279-019-01068-y

Veldman, S., Santos, R., Jones, R., Sousa-Sá, E. \& Okely,A. (2019). Associations between gross motor skills and cognitive development in toddlers. Early human development, 132, 39 44.https://doi.org/10.1016/j.earlhumdev.2019.04.005

Westendorp, M.,Hartman, E., Houwen,S., Smith,J., \&Visscher, C. (2011). The relationship between gross motor skills and academic achievement in children with learning disabilities. Research in developmental disabilities, 32(6), 2773-2779. https:/ /doi.org/10.1016/j.ridd.2011.05.032

Wick, K., Leeger-Aschmann, C.S., Monn, N. D., Radtke,T., Ott, L.V., Rebholz, C.E., . . \& Kriemler, S. (2017). Interventions to Promote Fundamental Movement Skills in Childcare and Kindergarten:A Systematic Review and Meta-Analysis. Sports medicine (Auckland, N.Z.), 47(10), 2045-2068. https:// doi.org/10.1007/s40279-017-0723-1

Yildirim, D., Bilge, M., \& Caglar, E. (2019). effects of a training program based on longitudinal training of athlete development (ltad) approach on gross motor skills among 6-8 years old children. European Journal of Physical Education and Sport Science, 5(3), 35-47.http/doi.org/10.5281/zenodo. 2532778

Zueck, M., Ramírez, A., Rodríguez, J., \& Irigoyen, H. (2019). Satisfacción en las clases de Educación Físicay laintencionalidad de ser activo en niños del nivel de primaria (Satisfaction in the Physical Education classroom and intention to be physically active in Primary school children). Retos, 37, 33-40. https:// doi.org/10.47197/retos.v37i37.69027 